\title{
Spatial interaction effects in letter processing
}

\author{
BO STRANGERT and LAURITZ BRÄNNSTRÖM \\ University of Umea, S-90187 Umea, Sweden
}

\begin{abstract}
The hypothesis of independent processing channels was tested in a letter-recognition task. Letter space and retinal position were varied for both unilateral and bilateral presentations of two-letter configurations. The response latencies indicated contour interference effects for small letter spaces as well as a different type of processing delay which increased rapidly with increased letter distance from the fixation point. The lat ter effect was assumed to represent an interaction between the feature extraction processes of the adjacent letters. Since the processing capacity decreases as a function of the distance from the fovea, the reaction times were correspondingly lengthened.
\end{abstract}

Selective visual attention controls the extraction of information during a single eye fixation. Mackworth (1965) described the useful field of view as the area around the fixation point where information is processed exhaustively. He suggested that the size of this area could be adjusted to prevent overload in the visual system, and he demonstrated that increasing amounts of peripheral visual noise made the field severely restricted. The role of peripheral vision in this respect is not clear. however, though the concept of the useful field of view should imply some form of low-level processing in the peripheral field.

If an array of letters is exposed so briefly that the subject has time only for a single fixation, he will probably process the array nonselectively during the early processing stages. The letter positions may be assumed to represent parallel input channels, and the clarity of information in a channel will be a function of is retinal position. Furthermore, the clarity of information should be a main determinant of the rate of information processing (Rumelhart, 1970). This simple relationship between processing rate and retinal locus may be modified by spatial conditions which affect the clarity of information. Thus, contour interference between adjacent letters in foveal vision has been estimated to occur when the spacing is about $1 / 3^{\circ}$ of visual angle or less (Eriksen \& Rohrbaugh, 1970; Flom, Weymouth, \& Kahneman, 1963). Naturally the interference effect is greater for embedded letters than for end letters of an array.

However, Eriksen and Hoffman (1972) found evidence for a spacing effect up to about $1^{\circ}$ of visual angle between adjacent letters and showed, furthermore, that this effect was significant only when both the target and noise elements were letters but not when a disk was used instead of a letter as noise. Since this result was difficult to reconcile with the contour interference hypothesis, they suggested an alternative explanation in terms of an attentional mechanism.

Requests for reprints should be sent to Bo Strangert, Comnunication Research Unit. University of Umeå, S-901 87 Umed. Sweden.
Thus. under normal conditions of viewing, the focus of the attentional field roughly corresponds to the foveal field which guarantees maximum clarity and fastest processing of information. But the attentional focus may be centered on perhaps any part of the visual field, thereby to some extent changing the distribution of information processing across the visual field. In the experiment by Eriksen and Hoffman, the spatial focus of attention was explicitly controlled by the presentation of a position indicator. We will assume that the subject can adjust his attentional focus, and hence change the spatial distribution of processing, also on the basis of information extracted during the early, low-level processing of the letter array.

Eriksen and Hoffman (1972) suggested that the attentional focus subtended only about $1^{\circ}$ of visual angle. According to the limited-capacity theory (Rumelhart. 1970), all elements falling within the attentional field would then have to share the processing energy that is available in that area. It is also necessary to specify the amount of processing needed to discriminate different types of elements, since the recognition of a target letter is impaired more by letter as noise than by other types of noise elements. Thus, the physical similarity or informational content of elements as well as their spatial proximity can be predicted to influence the processing rate.

In the present experiment, the space between two letters was systematically increased from one extreme condition of unilateral presentation where contour interference effects should be the essential cause of performance decrement, to the other extreme condition of bilateral presentation where the letter positions can be regarded as truly spatially independent processing channels. Provided that the letter space is just wide enough to eliminate most contour interference effects, the feature extraction processes of the adjacent letters could still interact. If the two letters cannot be encoded as a single meaningful pattern or chunk, then the speed of processing should be slower than in the case of widely 
Table 1

Twelve Examples of Stimulus Displays

\begin{tabular}{|c|c|c|}
\hline $\begin{array}{c}\text { Left Visual Field } \\
2.27 \mathrm{Deg} \\
\end{array}$ & $0 \mathrm{Deg}$ & $\begin{array}{c}\text { Right Visual Field } \\
2.27 \mathrm{Deg}\end{array}$ \\
\hline 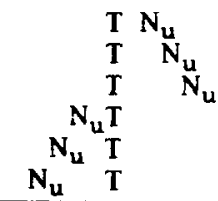 & & \begin{tabular}{cc}
\multicolumn{1}{c}{$\mathbf{N}_{\mathbf{b}}$} & \\
$\mathrm{N}_{\mathbf{b}}$ & \\
$\mathrm{N}_{\mathbf{b}}$ & \\
& $\mathbf{N}_{\mathbf{b}}$ \\
& $\mathrm{N}_{\mathbf{b}}$ \\
& $\mathbf{N}_{\mathbf{b}}$
\end{tabular} \\
\hline
\end{tabular}

Note-Targets at $2.27 \mathrm{deg}$ and the corresponding noise elements in the unilateral $\left(N_{u}\right)$ and bilateral $\left(N_{b}\right)$ conditions.

separate and independent channels. This prediction is based upon the assumption that the available processing capacity may be divided between the two parallel input channels.

\section{METHOD}

The present experimental technique involved a fixed sequence of visual events. starting with a brief exposure of a probe letter in the center of the visual field. A short interval later. the two stimulus letters were presented unilaterally or bilaterally in the exposure field. Finalls. a masking stimulus was exposed. The subject had time to fixate and identify the probe letter but not to make a new eve movement until after the stimulus array had been exposed. Thus. during a brief period, the subject was processing the stimulus letters while, at the same time. he had a memory representation of the probe letter.

\section{Stimulus Material}

A stimulus array consisted of two letters drawn randomly without replacement from the set of capital letters BCD FLMNPRSTV. Each array contained one target letter which matched the probe letter and whose left right position was determined randomly. All letters were used with equal frequency.

Fur the condition of unilateral presentation. two sets of stimulus arra! were constructed. In the tirst set. the target element could occupy either of two fixed positions at. respectively. $2.27^{\circ}$ and $3.37^{\circ}$ of risual angle from the central fixation point. For each fixed position. the space between the contour of the adjacent noise element and the target was varied in three levels (spacings. $.06^{\circ}$. $\left..43^{\circ} .80^{\circ}\right)$. The noise element occupied either a more peripheral or a more central position than the target (see the examples in Table 1).

In the second set. the noise element occupied one of the two fixed positions at $2.27^{\circ}$ and $3.37^{\circ}$. while the space to the target element was varied in a way similar to the first set. This arrangepent with two stimulus sets made it possible to explore the importance of the relative position of the target. The design also included a random variation of the left/right visual field. A total of 48 experimental conditions of unilateral presentation were formed.

For each unilateral condition. a corresponding bilateral preschution was constructed where one of the two letters was presented as in the unilateral set while the other letter was presented in the opposite hemifield but at a corresponding retinal distance from the fixation point (Table 1 ). The relative stimulus intensities were consequently the same as in a the unilateral condition. but the factor of spatial proximity was practically eliminated. Since the space varied between $3.13^{\circ}$ and $7.53^{\circ}$. no effects of spatial interaction on low-level processing could be expected.

The stimulus letters were constructed from a $5 \times 7$ dot matrix which gave letters of equal width $\left(.31^{\circ}\right)$ and height $\left(.42^{\circ}\right)$. The masking field was a random pattern of dots. $1.06^{\circ} \times 10.52^{\circ}$. constructed from the same type of matrix. Simultaneous exposures of the masking field and various stimulus configurations showed the masking effect to be complete even with extremely long viewing durations $(60 \mathrm{sec})$.

\section{Procedure}

The probe letter. the stimulus array, and the masking field were back-projected on a semitransparent screen, $130 \mathrm{~cm}$ in front of the eves of the subject. by means of a timer-governed projector system including electronic shutters. The stimuli appeared as bright. orange-colored figures against a dark background and the corresponding luminance values were: $1 \mathrm{~mL}$ (background). $20 \mathrm{~mL}$ (probe). $24 \mathrm{~mL}$ (stimulus letters), and $28 \mathrm{~mL}$ (masking stimulus). Viewing was binocular.

A trial involved the following events: Auditory forewarning signal (200 msec). silent interval $(400 \mathrm{msec})$, probe exposure $(40 \mathrm{msec})$. dark interal $1160 \mathrm{msec})$. stimulus exposure $(40 \mathrm{msec})$. dark interval $(230 \mathrm{msec})$, masking field (100 msec). A new trial started $3.5 \mathrm{sec}$ later. After each block of $\mathbf{4 8}$ trials. there was a pause of $1.5 \mathrm{~min}$.

The luminance values and the exposure durations were continuously recorded and controlled by the experimenter. By presenting white noise of $55 \mathrm{~dB}$ in the subject's earphones, the sound of the apparatus was masked. The onset of the stimulus exposure activated a photocell connected to an electronic counter (calibrated in milliseconds) which was stopped by the subject's manual choice reaction.

The subject was instructed to identify the probe letter and, at the time of stimulus exposure. to rapidly indicate, by pressing the respective left or right response key, whether the left or right letter matched the probe letter. (In the meantime, his index fingers rested on the keys that were attached to the arms of the chair.) In order to reduce the subject's response biases, he was fully informed in the practice session about the properties of the stimulus material and the randomness of presentation. A symmetric payoff matrix was used where correct responses were not rewarded but errors were penalized. Knowledge of the frequency of errors was given after each block together with a summary statement of the mean and distribution of RTs.

The experiment included one practice session and three experimental sessions. An experimental session began with 24 practice trials. which were followed by 12 replications of the basic design with the 96 conditions. The conditions were ordered randomly within blocks of 96 trials, and each subject received the 12 blocks in a unique order.

\section{Subjects}

One female and five male university students served as subjects and were paid $100 \mathrm{Skr}$ (approximately $\mathbf{5 2 0}$ ) and an additional bonus of maximum $5 \mathrm{Skr} /$ session. All were right-handed and had normal vision.

\section{RESULTS}

The combined data from the three experimental sessions yielded both small frequencies and short reaction times. on average. For unilateral presentation. the mean proportion of incorrect responses was .058 , while for bilateral presentation it was less than .003 . The mean difference between the lett and right visual field was .001. Incorrect responses occurred predominantly in the condition where the target letter occupied an extreme peripheral position $\left(>3.73^{\circ}\right)$. There the error proportions were $.24 . .21$. and .19 at the spacings of $.06^{\circ} . .43^{\circ}$. and $.80^{\circ}$, respectively. When. instead. the noise element occupied the extreme peripheral position, the corresponding error proportions were $.13 . .06$. and 


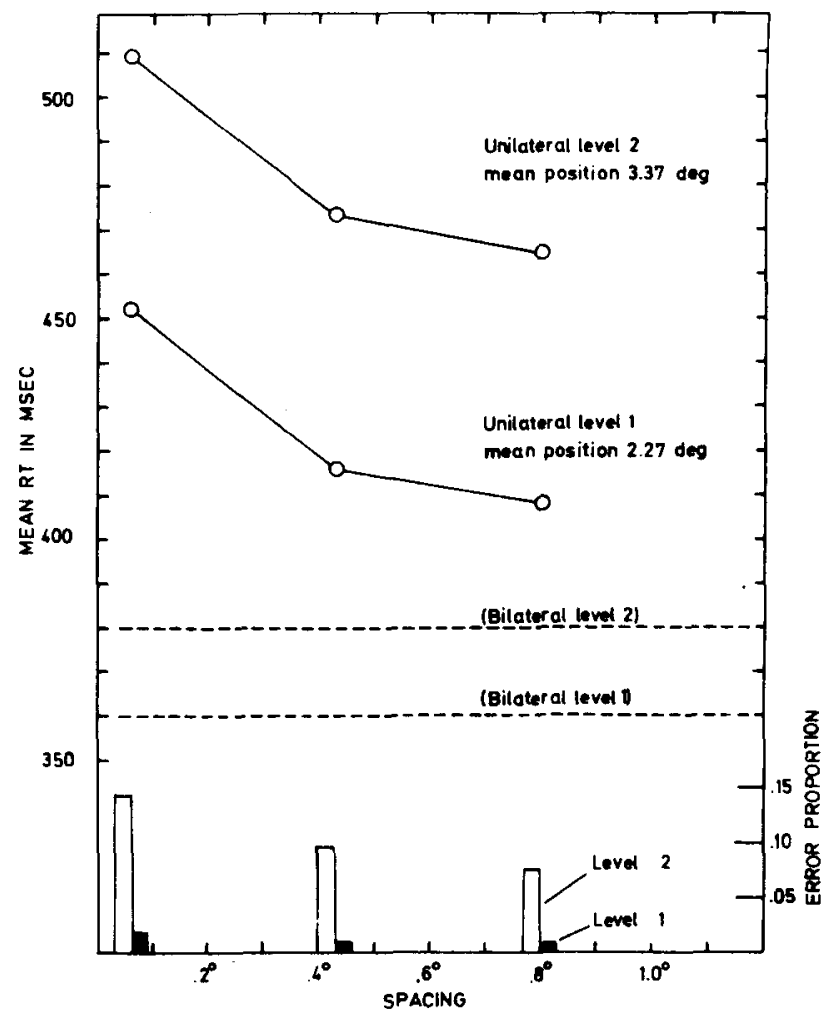

Figure 1. Mean RT of correct responses and mean proportion of errors as a function of spacing for two levels of retinal position in the unilateral condition.

.03. This is the only result which is inconsistent with the hypothesis that the target and the noise element were processed equivalently and to the same degree.

Arithmetic mean RTs of correct responses were computed for all conditions and subjects. The difference in mean RT between the left $(455 \mathrm{msec})$ and the right visual field $(450 \mathrm{msec})$ for unilateral presentation was definitely not significant. For bilateral presentation, the mean RTs were 369 (LVF) and 370 (RVF) msec. Furthermore, the overall mean difference between the left and right response hand was only $1.0 \mathrm{msec}$. Since neither the factor of visual field nor that of response hand interacted with any other independent variable, the data for those factors were combined.

Are the target and the noise letter processed equivalently and to the same degree? Partial information concerning this question may be obtained by assessing the effect of the relative target position in the array. The results indicate that configurations with the target in a more peripheral position than the noise letter delayed the reactions by only $1.1 \mathrm{msec}$ (bilateral presentation) and $1.3 \mathrm{msec}$ (unilateral presentation), on the average, compared to configurations with the reverse positions of elements. This insigniticant difference is valid for all conditions except for the single cases of unilateral presentation where the target was in an extreme position $\left(>4.10^{\circ}\right)$ and accordingly supplied very little information (cf. the error data). There the reactions on the average were delayed by about 20 msec compared to the case with the noise element in the extreme periphery. The general conclusion is that the relative position of the target within the letter array was of no importance for almost all of the conditions studied. Consequently, the two elements seem to have been processed equivalently and the retinal positions of both letters nust be specified in order to predict the array information and its effect on processing rate.

The occurrence of contour interference is indicated by the signilicant spacing effect for both levels of retinal position in the unilateral condition, $F(2.5)=$ $30.74, p<.01$ (Figure 1). When the letter space is $.06^{\circ}$, the mean $R^{\top} T$ is significantly longer than at $.43^{\circ}$ $(p<.01)$. but with increased spacing to .80 the further improvement is small and insignificant $(p>.05)$, as revealed by comparisons between individual means. Since the error frequency was quite large and the error latencies were generally long at the second level of retinal position, the corresponding mean RT's of correct responses may be spuriously short compared to those of the other spacing conditions. Bilateral presentation eliminates the effect of spatial proximity and gives very small variations in mean R'T which can be attributed to differences in the position of the letter array (Table 2).

The basic factor affecting the processing rate is thus the retinal positions of the elements. As expected, the mean RT increases signiticantly with increased letter distance from the fixation point $[F(1.5)=34.83$, $p<.011$. and this is valid for variations in the fixed level of both the target and the noise element and for both unilateral and bilateral presentation. Figure 2 show's the mean difference in RT between the unilateral conditions and their bilateral counterparts as a function of the mean distance of the two letters from the fixation point. The mean visual angle was

Table 2

Mean RT of Correct Responses for Unilateral and Bilateral (Within Parentheses) Conditions

\begin{tabular}{|c|c|c|c|c|c|c|}
\hline \multirow{2}{*}{$\begin{array}{c}\text { Mean } \\
\text { Position } \\
\text { (Deg) }\end{array}$} & \multicolumn{6}{|c|}{ Unilateral Spacing (Deg) } \\
\hline & & 6 & & 3 & & 30 \\
\hline $\begin{array}{r}\text { Level 1 } \\
1.72 \\
1.90 \\
2.08\end{array}$ & 453 & $(358)$ & 405 & (358) & 393 & (358) \\
\hline $\begin{array}{l}2.46 \\
2.64 \\
2.82\end{array}$ & 450 & $(358)$ & 426 & $(366)$ & 422 & (359) \\
\hline $\begin{array}{r}\text { Level } 2 \\
2.82 \\
3.00 \\
3.18\end{array}$ & 506 & $(381)$ & 455 & (374) & 433 & (373) \\
\hline $\begin{array}{l}3.56 \\
3.74 \\
3.92\end{array}$ & 512 & $(378)$ & 491 & $(381)$ & 487 & (386) \\
\hline
\end{tabular}




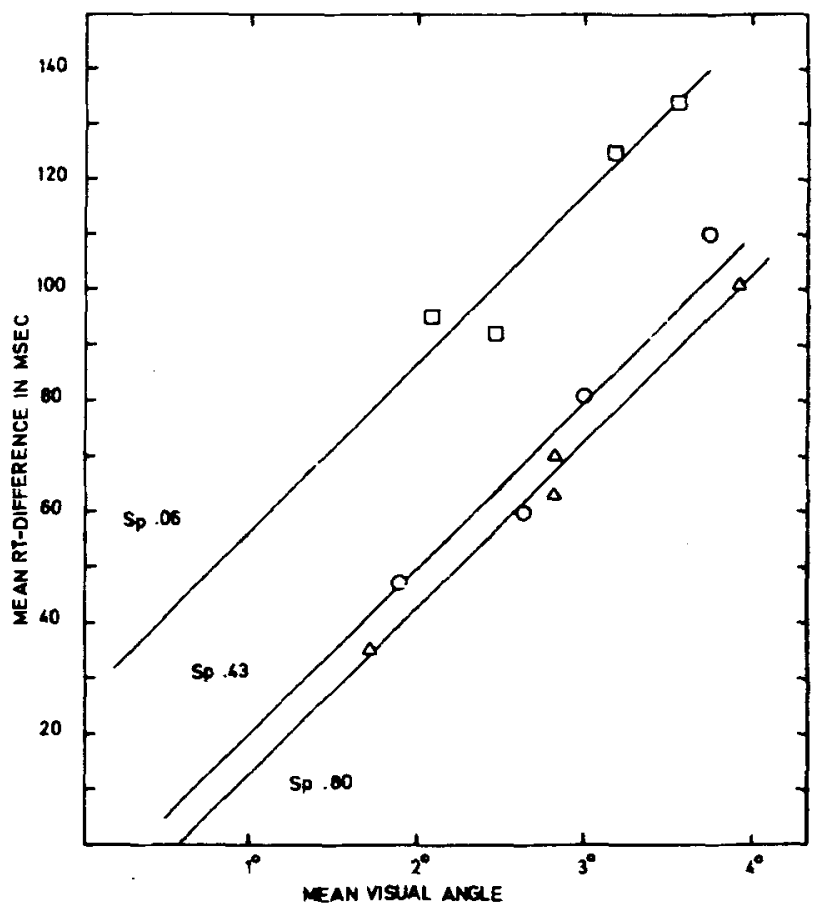

Figure 2. Mean RT difference between the unilateral and bilateral conditions as a function of array position for three lesels of unilateral spacing.

used as a correlate of the intensity value of the letter array. Since the corresponding unilateral and bilateral conditions are similar except for the spacing factor. the mean RT difference should reflect the processing delay caused by spatial interaction between the channels. Two distinct effects are evident in Figure 2. The intercept differences between the three titted parallel lines reflect the spacing effect due to contour interference. There are some minor. systematic deviations from the line of spacing .43 . suggesting that the estimated parameters must be regarded with some caution. The equations are: $\mathrm{RT}(.06)=30 \mathrm{p}+27 ; \mathrm{RT}(.43)=30 \mathrm{p}-10 ;$ $\mathrm{RT}(.80)=30 \mathrm{p}-17$. Linear regression accounts for $92 \%, 96 \%$, and $99 \%$, respectively, of the variation of the mean RT as a function of mean position (p).

The most important aspect of the data in Figure 2 is the interaction between the laterality factor and the retinal position which is expressed by the striking effect on unilateral mean RT of increased letter distance from the fovea. Since this effect is roughly similar for the three spacing levels, it is inconsistent with an explanation in terms of contour interference but consistent with the hypothesis of spatial interaction between feature processing channels.

\section{DISCUSSION}

The difterence between the unilateral and bilateral conditions in the mean slope of the RT functions is a clear expression of an interaction contingent on the spatial proximity of unconnected letters. The results are thus in general agreement with the predictions based on linited-capacity theory and the findings of Eriksen and Hoftman (1972). Before this theoretical line is further discussed, a few alternative interpretations of the data will be examined.

The first alternative is that the difference between the unilateral and bilateral conditions is due mainly to contour interference. The curves in Figure 1 reveal the decreasing effect of contour interference with increased spacing. But extrapolating the curves down to the vicinity of the bilateral $\mathrm{RT}$ levels gives estimates of separation which are clearly incompatible with the contour interference phenomenon. At least for the data of Level 1. it seems unreasonable to extend the region of interference much beyond the largest spacing of .80 used in the present experiment. As for the data of Level 2. however. the error proportions were quite large. Since the RTs were generally longer for incorrect than for correct responses, it is possible that the mean RTs of correct responses are spuriously short. Thus. the contour interference effect may have been underestimated for the small spacings and peripheral positions. That may explain why the expected covariation between contour interference and retinal position is missing for the data (cf. Alpern. 1953). Consequently, the approximate parallelism of the functions in Figure 2 may be a coincidence; at least for the spacing .06 and. to a less degree, for the spacing .43 , the slopes should be steeper. However. these qualifications seem to have little bearing upon the substantial mean RT differences between the bilateral and unilatera: conditions. e.g., for the spacing of $.80^{\circ}$.

Another conceivable interpretation of the laterality difference might be in terms of response compatibility. In the bilateral conditions, the hemitield factor and the target position are perfectly correlated and coupled with a fixed response finger. while the unilateral presentation in half the cases requires less compatible responses, e.g., the target in a right position in the left visual field, requiring a right-linger response. This should lead to delayed responses and eventually more errors for targets occupying a more central position than the noise element. As a matter of fact. there was practically no effect of relative target position, but on the basis of the present data it is impossible to exclude a difference in response compatibility. However, essentially the same general results were recently obtained in a similar experiment by Brännström (Note 1), who used an entirely different response method. a yes/no paradigm, and studied accuracy by SDT measures. This fact certainly makes the compatibility hypothesis seem less adequate. The similarity of results in spite of the different response methods also makes another hypothesis seem unlikely. namely that the use of left/right responses could lead the subject in the bilateral condition to 
select only one processing channel and then directly decide upon the response. This seems unlikely also with respect to the very small means and variances of the unimodal $R T$ distributions in the bilateral condition.

Thus. even taken together, the alternative interpretations do not seem sufficient to handle the large laterality difference. On the other hand, the results contirm the hypothesis of a spatial interaction between the adjacent feature processing channels in the unilateral conditions. According to the assumptions made, this spatial interaction may be interpreted as a kind of interference or nonindependence between the feature processing channels. i.e.. the processing of the individual letters is delayed by the conflicting use of common feature analyzers or a common amount of processing energy. (Alternatively. the adjacent elements can just às well be assumed to be processed within one and the same channel.) That should be a hindrance only insofar as the letters are unconnected; should they form a meaningful pattern, then their spatial proximity would instead allow more efficient higher level processing units to operate. The factor of spatial selectivity sets a boundary on the interaction effects. In terms of retinal distance, the effects obviously extend somewhat beyond the largest spacing of $.80^{\circ}$ used in the unilateral conditions. The general implication is that the more elements or process details within a restricted area, the more the common processing capacity is used up, with slower processing rate as a consequence. Since the quality of the sensory information, as well as, probably, the effectiveness of the feature analyzers. is reduced monotonically as a function of the distance from the fixation center, the net effect will be that the processing rate varies concomitantly.

The results are thus consistent with the basic assumptions. namely that the attention or available processing capacity may be divided between the two processing channels. that these channels are functionally parallel, and finally, that the channels are spatially independent in the bilateral condition but interfering in the unilateral condition. However, it is possible to relax the assumption of active interference and at the same time specify some possible locations of capacity limitations. Thus, on a low level of visual processing. the notion of limited capacity could mean a property of a restricted visual area. Consequently, the processing of closely spaced elements will be delayed simply due to the limited local channel capacity. It is further reasonable to assume that the local processing capacity cannot be redistributed freely across the visual field and also that there must be a limit to the number of processing chamnels that may work in parallel. This moditied hypothesis of spatial interaction is consistent with the obtained RT difference between the bilateral and unilateral conditions. But only further research may permit the evaluation of this and various other hypotheses. For example, a most provocative alternative hypothesis is that the spatial interaction effect is not related to the level of feature extraction but depends on a more complicated matching between the probe and the extracted letter patterns.

\section{REFERENCES}

At.PERN, M. Metalcontrast. Jommal of the Optical Society "I America. 1453. 43. 648-657.

Eriksfan, C. W.. \& Hoffman, J. E. Temporal and spatial chatacteristics of selective encoding from visual displays. Preceltion \& Psychophysics, 1972, 12, 201.204.

Eriksen. C. W.. \& Rohrbaugh. J. W. Sone factors determining efficiency of selective attention. American Journal of Psychology'. 1970 . 83. 330-342.

Flom. M. C., Weymouth, F. W., \& Kahneman, D. Visual resolution and contour interaction. Journal of the Optical Sociely of America, 1\%63, 53, 1026-1032.

Mackworth, N. H. Visual noise causes tunnel vision. Psychonomic Science. 1965, 3, 67-68.

RUmelhart. D. E. A multicomponent theory of the perception of brietly exposed visual displays. Journal of Mathematical Psychology, 1970, 7, 191-218.

\section{REFERENCE NOTE}

1. Brännström, L. Effects of spatial factors upon accuracy of visual information processing. Project Report. November 1973, Communication Research Unit. University of Umea.

(Received for publication September 6. 1974: revision received November 18.1974.$)$ 